\title{
The Utility of Next-Generation Sequencing in the Treatment Decision-Making for Metastatic Non- Small-Cell Lung Cancer
}

Maria Cristina Orlov-Slavu ${ }^{1}$, Ana Maria Popa ${ }^{1}$, Adrian Tulin ${ }^{2,} 3$, Anca Pantea Stoian ${ }^{4}$, Catalina Poiana ${ }^{5}$, Cristian Paleru ${ }^{6}$, Valentin Calu ${ }^{7}$, Cornelia Nitipir ${ }^{1}$

1. Oncology Department, Carol Davila University of Medicine and Pharmacy, Bucharest, ROU 2. General Surgery, Agrippa Ionescu Emergency Hospital, Bucharest, ROU 3. Human Anatomy Department, Carol Davila University of Medicine and Pharmacy, Bucharest, ROU 4. Diabetes, Nutrition and Metabolic Diseases Department, Carol Davila University of Medicine and Pharmacy, Bucharest, ROU 5. Endocrinology, National Institute of Endocrinology C.I.Parhon, Bucharest, ROU 6. Thoracic Surgery, Marius Nasta National Institute of Pneumology, Bucharest, ROU 7. General Surgery, Elias Emergency Hospital, Bucharest, ROU

Corresponding author: Ana Maria Popa, ana-maria.popa@umfcd.ro

\section{Abstract}

Next-generation sequencing (NGS) is a fast and relatively inexpensive method to sequence a large number of genes with crucial importance in cancer medicine. Nowadays, NGS is frequently used in diagnostic and therapeutic decisions in oncology; however, recently, it was demonstrated that only a few cancer sites actually benefit from this assessment. Moreover, the association of a mutant gene with a targeted drug is not always as predicted during in-vitro trials and is often not associated with tumor response. To predict the efficacy of such an association several classification systems have been developed. The present review aims to analyze the most important tumor agnostic treatment trials and assess how they shape selecting cancer patients for NGS. Moreover, it aims to determine how mutation-drug associations can be classified by their targetability and level of evidence of efficacy in non-small-cell lung cancer.

Review began 07/16/2021 Review ended 07/29/2021 Published 08/05/2021

\section{๑) Copyright 2021}

Orlov-Slavu et al. This is an open access article distributed under the terms of the Creative Commons Attribution License CC-BY 4.0., which permits unrestricted use, distribution, and reproduction in any medium, provided the original author and source are credited.
Categories: Oncology, Pulmonology

Keywords: cancer genomic profiling, targeted therapy, oncology, tumor agnostic treatment, next-generation sequencing

\section{Introduction And Background}

Next-generation sequencing (NGS) is the most accessible modality to describe genomic alterations in patients with cancer from both tumor tissue samples and circulating cell-free DNA. The characteristics of each tumor and possible drug targets can be determined using NGS. Tumor tissue samples can be inadequate for biomarker analysis because the DNA extracted is insufficient and/or degraded. To overcome these limitations, researchers have explored the possibility of using liquid biopsy as an alternative method for biomarker testing. The possibility to personalize oncological treatment is thought to lead to superior efficacy than classical drug targets. However, detecting targetable mutations is not always followed by therapeutic success, and the same drug may not have the same results in different cancer sites and histologies [1].

In order not to use these costly genetic assessments in vain, the classification of genetic alterations by their targetability has been proposed. Practicing oncologists report low self-confidence when choosing a type of drug-alteration association, as has been reported in the literature [2].

The present review aims to describe the most important trials centered on personalized oncology and tissue agnostic therapies, to describe how eligible patients for these tests can be selected, and to determine how one alteration and its correspondent drug can be classified according to the targetability and level of evidence of efficacy in non-small-cell lung cancer (NSCLC).

\section{Review}

\section{Insights gained from important precision medicine trials}

We consider the National Cancer Institute-Molecular Analysis for Therapy Choice (NCI-MATCH) and SHIVA (molecularly targeted therapy based on tumor molecular profiling versus conventional therapy for advanced cancer) to be the landmark trials for histology agnostic cancer treatment. These trials have shaped how we view precision medicine today $[3,4]$.

To better illustrate this some of the data presented in these two trials will be presented here. In the NCIMATCH trial, 65 patients with 45 different histologies were selected to receive the phosphoinositide 3-kinase (PI3K) inhibitor taselisib. All of the selected patients had the phosphatidylinositol-4,5-bisphosphate 3-kinase catalytic subunit alpha (PIK3CA) activator mutation, but no tumor response was recorded [5]. In addition, 
patients with solid tumors or lymphomas harboring BRAF mutations other than the well-known V600E did not benefit from trametinib treatment in the same trial [6]. Moreover, when all human epidermal growth factor receptor 2 amplified gene patients (except gastric and breast cancer patients) were treated with adotrastuzumab emtansine, no benefit was reported [7].

Second, we consider the data from the SHIVA trial, the first prospective trial that evaluated the efficacy of NGS-selected oncology treatments in heavily treated patients with different cancer sites. The trial enrolled 195 patients with alterations in rapidly accelerated fibrosarcoma-mitogen-activated protein kinase, PI3K, and hormonal receptor signaling pathways. The patients were randomized according to physician choice of cytotoxic chemotherapy or targeted treatment. The progression-free survival (PFS) was not superior in the experimental arm (2.3 months versus 2 months; hazard ratio $=0.88$, confidence interval $=0.65-1.19, \mathrm{p}=$ 0.41) [4].

Finally, the insight from these data can be summarized as the following: there was no reliable differentiation between the supposed driver mutations and other possible actionable mutations and the design of these trials was unidirectional and took into account only one treatment-alteration association. It would be ideal to analyze the entire mutational landscape, discuss the significance of all alterations, and prioritize their targeting. Even if not all tested patients have targetable mutations, the global data from this analysis can be very helpful in cancer research. These trials not only teach how to design future tumor agnostic therapy studies but also shape the way we view mutation targetability in general [8].

\section{Tools to aid decision-making in precision medicine}

To avoid these problems, classifications were developed to aid physicians in selecting targeted therapies for the alterations. These classifications organize the current knowledge about the efficacy of drugs in different alterations and histologies. All of them describe the antitumor effect analyzing the magnitude of clinical benefit. Chronologically, the first classification was published in 2014 by Van Allen et al. and included five levels of evidence that reported the predictive, prognostic, and diagnostic value of the biomarkers used to select therapies [9]. In 2015, Dienstmann et al. proposed a different ranking method that considered the genetic abnormality, the variant, and the cancer site in which it can be found and reported the magnitude of clinical benefit considering each of these [10]. In 2016, Sukhai et al. focused on somatic variants and proposed classification included the ability to be a driver mutation, the site it can be found in, and the harboring histologies [11].

The first ranking that related possible drugs with their level of evidence and how they are perceived in international guidelines, especially the National Comprehensive Cancer Network was published in 2017 and was called OncoKB. This classification includes the alteration-drug associations categorized into four levels of evidence and ranks them according to their acknowledgment by decision agencies, such as the Food and Drug Administration. In addition, it also takes into consideration that targeting one association does not have the same impact in different histologies, that some genetic abnormalities are predictive of resistance, and that some oncogenic mutations cannot be targeted [12].

However, the most used ranking system is the ESMO Scale for Clinical Actionability of molecular Targets (ESCAT) which links the genetic alteration to its clinical relevance. When establishing in which tier to classify a drug, clinical trials are analyzed for statistical significance. One example for different histologies is presented in Table 1 . Tier I includes associations that are supported by proof from clinical trials and can be considered as the standard of care. Tiers IA, IB, and IC can be defined as the following: IA, randomized clinical trials that proved a benefit in survival; IB: randomized clinical trials that reported clinically meaningful benefit; and IC, efficacy proven in basket trials. 


\section{Cureus}

\begin{tabular}{|c|c|c|c|}
\hline Tier level & Alteration & Tumor site & Drug used \\
\hline IA & BRCA 1/2 germline mutation & Breast cancer & Talazoparib [13] \\
\hline IB & FGFR fusion & Cholangiocarcinoma & Pemigatinib [14] \\
\hline IC & NTRK fusion & Breast cancer, colorectal cancer, gastric cancer & Entrectinib $[15,16]$ \\
\hline IIIA & PTEN deletion or mutation & Prostate adenocarcinoma & Ipatasertib(+abiraterone) $[17,18]$ \\
\hline IIIB & AKT1 mutation & Breast cancer & AZD5363 [19] \\
\hline IIIA & PIK3CA hotspot mutation & Hepatocellular carcinoma & Alpelisib [20] \\
\hline IIIIB & ERBB3 mutation & Breast cancer, gastric cancer & Neratinib [21] \\
\hline
\end{tabular}

\section{TABLE 1: ESCAT ranking in other histologies than NSCLC.}

AKT1: AKT serine/threonine kinase 1; ERBB3: Erb-b2 receptor tyrosine kinase 3; ESCAT: ESMO Scale for Clinical Actionability of molecular Targets; FGFR: fibroblast growth factor receptor; NSCLC: non-small-cell lung cancer; NTRK: neurotrophic tyrosine kinase; PIK3CA: phosphatidylinositol-4,5bisphosphate 3-kinase catalytic subunit alpha; PTEN: phosphatase and tensin homolog

Tier II describes associations that were reported as significant but are not sustained by prospective studies. When deciding to treat with such an association, the drug should be given in an access program or in an ongoing prospective trial. Tier IIA includes associations that were proven to be beneficial in retrospective studies, and IIB includes associations that cannot be subjected to a prospective analysis. Tier III includes alterations whose importance has been proven for other histologies than the one in question. In IIIA, the benefit has already been proven in other tumor types, whereas IIIB describes an association with an extremely probable clinical benefit with no proof of efficacy yet (for no histology). Tier IV describes genetic abnormalities that have been proven to be targetable in preclinical trials. Tier IVA includes proof from invitro studies or in-vitro cancer models, and IVB includes predictions of efficacy derived from informatic systems. Tier X includes all abnormalities that are known to be untargetable or have never been proven beneficial when associated with drugs. However, such abnormalities should not be permanently considered part of this subgroup (they could prove useful when associated with a different alteration or when tested with different technology) [22].

\section{Next-generation sequencing for non-small-cell lung cancer: when and where?}

The latest recommendations for NGS testing made by the European Society of Medical Oncology) list NSCLC as the only cancer in which it makes sense to use multigenic panels that include all alterations with ESCAT I level of targetability. Given that gene fusions are very important in this cancer site, the NGS panels used are of the DNA or the RNA type. The most important mutations and fusions are illustrated in Figure 1. 


\section{Cureus}
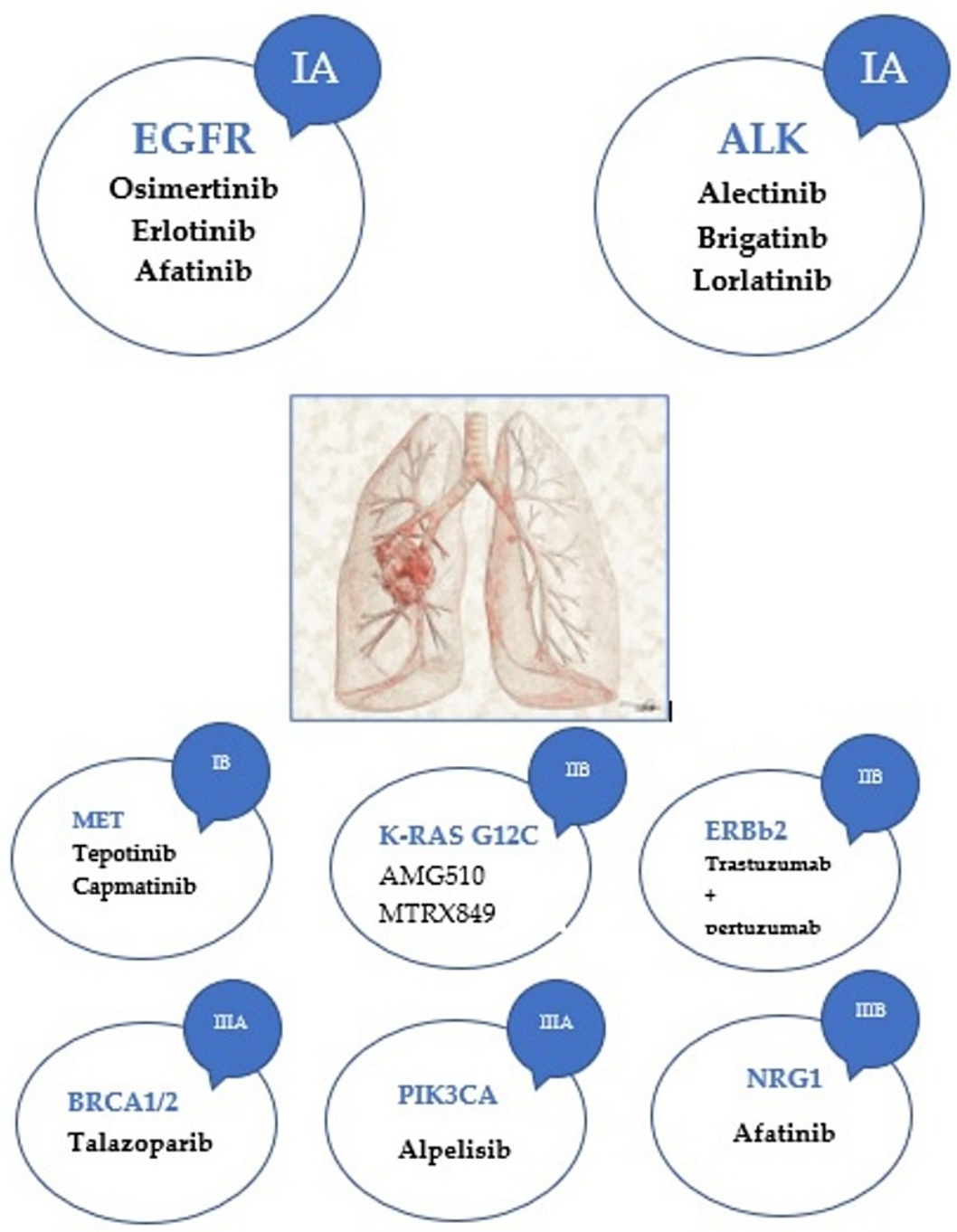

FIGURE 1: Genetic alterations in NSCLC and their level of evidence for targetability according to ESCAT

ALK: anaplastic lymphoma kinase; EGFR: epidermal growth factor receptor; ERBb2: erythroblastic oncogene B; ESCAT: ESMO Scale for Clinical Actionability of molecular Targets; K-RAS: Kirsten rat sarcoma oncogene; NRG1: neuregulin 1 gene; NSCLC: non-small-cell lung cancer; PIK3CA: phosphatidylinositol-4,5bisphosphate 3-kinase catalytic subunit alpha

For global benefit, the tests for alterations with evidence lower than ESCAT 1 have debatable significance. They can be considered if the patient is negative for frequent alterations, the healthcare system in the country has a reimbursement plan for off-label drugs, or if there is an early access program in which patients can be enrolled to receive them [23].

The most common alterations in NSCLC are the well-known epidermal growth factor receptor (EGFR) mutation and anaplastic lymphoma kinase ( $A L K$ ) fusion. Within the different subtypes of EGFR mutations, the levels of evidence for targetability vary. The most common driver mutations, namely, exon 19 deletion and exon 21 (L858R) substitution (15\% of the general population and $50-60 \%$ of the Asian population), are associated with the most data. To these, the mutation T790M in exon 20 is added, which is usually acquired and marks resistance to treatment with older generation tyrosine kinase inhibitors such as erlotinib $[24,25]$.

All these alterations are classified as ESCAT I, and the preferred treatments in these circumstances include osimertinib, erlotinib, afatinib, and gefitinib. Most countries reimburse at least one of these treatments as the first line in these patients [26-29]. However, there are EGFR mutations with a lower level of targetability such as $G 719 \mathrm{X}$ mutation in exon $18, S 768 I$ in exon 20, and $L 861 \mathrm{Q}$ in exon 21. The prevalence of these mutations in NSCLC is 10\% and can be classified as ESCAT IIB. Prospective, nonrandomized studies have shown benefit in at least PFS when patients with rare EGFR mutations were treated with osimertinib and afatinib [26,30-32]. 
Insertions in exon 20 , with a prevalence of approximately $2 \%$ in NSCLC, are considered predictive of the lack of response to classical tyrosine kinase inhibitors used in patients with EGFR mutations. However, there is currently a therapeutic option in this situation: poziotinib. An ongoing phase 2 trial has already reported a response rate of approximately $64 \%$ with this treatment in patients with exon 20 EGFR insertions. ESCAT ranking for this alteration remains open until further data are obtained [33].

The next gene alteration in prevalence and importance in NSCLC is $A L K$ fusion (5\%), classified as ESCAT IA. The preferred treatment in this situation includes alectinib, brigatinib, or lorlatinib [34-36].

Among the alterations with a lower level of evidence for targetability, we mention $M E T, B R A F$, neurotrophic tyrosine kinase (NTRK), rearranged during transfection (RET), Kirsten rat sarcoma oncogene (K-RAS), ROS, erythroblastic oncogene B (ERBB2), BReast CAncer gene (BRCA1/2), PIK3CA, and neuregulin 1 gene (NRG1). Phase I and II clinical trials have confirmed clinical benefit in targeting and improving overall response rate [23].

The next alteration worth reviewing is $M E T$ exon 14 skipping mutation considered targetable with crizotinib, capmatinib, and tepotinib, with the latter two being considered by current guidelines as preferred treatments. It has a level of evidence Ib and a frequency of 3\% in NSCLC [23,37-39].

$K-R A S$ can also be classified among the important mutations in NSCLC, being a relatively frequent mutation (12\%). It has a level IIB targetability [23]. The most druggable subtype of this mutation is K-RAS G12C (substitution of glycine 12 to cysteine). Trials are underway with several drugs such as AMG510 (Amgen) and MTRX849 (Mirati Therapeutics) in both monotherapy or combined with immunotherapy [40,41]. In addition, some phase I trials with mature data included older drugs for $K$-RAS mutation. Two worth mentioning are sorafenib, a multikinase inhibitor that targets upstream of the substrate of this mutation combined with the mTOR inhibitor (mammalian target of rapamycin) and everolimus. Unfortunately, this combination was not enough to induce a lasting tumor response [42,43].

Among the alterations with lower levels of targetability in NSCLC, but worthy of special attention, are ESCAT IIB level: ERBB2 (amplifications or hotspot mutations); ESCAT IIIA: BRCA1/2 and PIK3CA (low frequency, approximately 1.2\%); and NRG 1-ESCAT fusion IIIB [23]. The most important alterations in NSCLC and the proposed targeting drugs are illustrated in Figure 1.

\section{The non-small-cell lung cancer issue: cost-effectiveness}

Broad NGS testing is the most cost-effective in NSCLC of all histologies $[44,45]$. To analyze the financial aspects of NGS in NSCLC, two trials were selected with similar endpoints but different populations. The first one, by Mateo et al., included 174 patients with metastatic NSCLC and tested them for 29 DNA alterations using an NGS panel (the panel included $E G F R, B R A F, E R B B 2, T P 53$ ) and three ARN fusions, namely, ALK, $R O S, R E T$. It aimed to determine the cost-effectiveness by analyzing the proportion of patients who would not have had detected the gene alteration had it not been for this test by reporting the actual financial burden and the time needed until the results were available [46]. However, the data can be considered biased as patients were only of Singaporean nationality, for which the percentage of EGFR-positive patients is higher than that for other populations. The trial concluded that NGS is a cost-efficient method to decide treatment in metastatic NSCLC and supported the implementation of NGS as a standard in the Asian population [46].

However, when NGS was compared with the classic sequencing test (the one including only EGFR mutations, $A L K$ translocation, and programmed death-ligand 1), only $1 \%$ of patients benefited from the additional detection of alterations $[47,48]$.

Steuten et al. compared the cost-effectiveness and clinical efficacy of single genetic marker tests with the determination of classical alterations in metastatic NSCLC by NGS. Cost-effectiveness was classified as moderate (after analyzing the cost of genetic tests, the frequency of targetable alterations, and the availability of correspondent therapies). Of the 5,688 patients studied, only 875 were tested by NGS. Overall, $21 \%$ received targeted treatment compared to $19 \%$ of the patients who underwent classical determination [49].

Overall, both trials reported moderate cost-effectiveness in the use of NGS in advanced NSCLC. To this must be added the default longer time to results. In addition, and most importantly, the use of NGS may lead to a situation where the patient has, according to the literature and guidelines, the indication of a targeted treatment, but it is not reimbursed in the country. These situations must be foreseen by public health forums of each country, eventually proposing a customized solution.

\section{Conclusions}

To conclude, NGS testing in NSCLC for ESCAT IA genetic alterations is of paramount importance and should be considered standard. However, alterations with lower evidence targetability should always be considered, 
with careful consideration given to the financial and medical aspects involved.

\section{Additional Information \\ Disclosures}

Conflicts of interest: In compliance with the ICMJE uniform disclosure form, all authors declare the following: Payment/services info: All authors have declared that no financial support was received from any organization for the submitted work. Financial relationships: All authors have declared that they have no financial relationships at present or within the previous three years with any organizations that might have an interest in the submitted work. Other relationships: All authors have declared that there are no other relationships or activities that could appear to have influenced the submitted work.

\section{Acknowledgements}

Cristina Orlov-Slavu and Adrian Tulin have the same contribution to this paper and should be regarded as first authors.

\section{References}

1. Trédan O, Wang Q, Pissaloux D, et al.: Molecular screening program to select molecular-based recommended therapies for metastatic cancer patients: analysis from the ProfiLER trial. Ann Oncol. 2019, 30:757-65. 10.1093/annonc/mdz080

2. Gray SW, Hicks-Courant K, Cronin A, Rollins BJ, Weeks JC: Physicians' attitudes about multiplex tumor genomic testing. J Clin Oncol. 2014, 32:1317-23. 10.1200/JCO.2013.52.4298

3. Flaherty KT, Gray RJ, Chen AP, et al.: Molecular landscape and actionable alterations in a genomically guided cancer clinical trial: National Cancer Institute Molecular Analysis for Therapy Choice (NCI-MATCH). J Clin Oncol. 2020, 38:3883-94. 10.1200/JCO.19.03010

4. Le Tourneau C, Delord JP, Gonçalves A, et al.: Molecularly targeted therapy based on tumour molecular profiling versus conventional therapy for advanced cancer (SHIVA): a multicentre, open-label, proof-ofconcept, randomised, controlled phase 2 trial. Lancet Oncol. 2015, 16:1324-34. 10.1016/S14702045(15)00188-6

5. Krop IE, Jegede O, Grilley-Olson JE, et al.: Results from molecular analysis for therapy choice (MATCH) arm I: taselisib for PIK3CA-mutated tumors. J Clin Oncol. 2018, 36:101. 10.1200/JCO.2018.36.15_suppl.101

6. Johnson DB, Zhao F, Noel M, et al.: Trametinib activity in patients with solid tumors and lymphomas harboring BRAF non-V600 mutations or fusions: results from NCI-MATCH (EAY131). Clin Cancer Res. 2020, 26:1812-9. 10.1158/1078-0432.CCR-19-3443

7. Jhaveri KL, Wang XV, Makker V, et al.: Ado-trastuzumab emtansine (T-DM1) in patients with HER2amplified tumors excluding breast and gastric/gastroesophageal junction (GEJ) adenocarcinomas: results from the NCI-MATCH trial (EAY131) subprotocol Q. Ann Oncol. 2019, 30:1821-30. 10.1093/annonc/mdz291

8. Pestana RC, Sen S, Hobbs BP, Hong DS: Histology-agnostic drug development - considering issues beyond the tissue. Nat Rev Clin Oncol. 2020, 17:555-68. 10.1038/s41571-020-0384-0

9. Van Allen EM, Wagle N, Stojanov P, et al.: Whole-exome sequencing and clinical interpretation of formalinfixed, paraffin-embedded tumor samples to guide precision cancer medicine. Nat Med. 2014, 20:682-8. 10.1038/nm.3559

10. Dienstmann R, Jang IS, Bot B, Friend S, Guinney J: Database of genomic biomarkers for cancer drugs and clinical targetability in solid tumors. Cancer Discov. 2015, 5:118-23. 10.1158/2159-8290.CD-14-1118

11. Sukhai MA, Craddock KJ, Thomas M, et al.: A classification system for clinical relevance of somatic variants identified in molecular profiling of cancer. Genet Med. 2016, 18:128-36. 10.1038/gim.2015.47

12. Chakravarty D, Gao J, Phillips SM, et al.: OncoKB: a precision oncology knowledge base . JCO Precis Oncol. 2017, 2017:PO.17.00011. 10.1200/PO.17.00011

13. Litton JK, Rugo HS, Ettl J, et al.: Talazoparib in patients with advanced breast cancer and a germline BRCA mutation. N Engl J Med. 2018, 379:753-63. 10.1056/NEJMoa1802905

14. Vogel A, Sahai V, Hollebecque A, et al.: LBA40 - FIGHT-202: a phase II study of pemigatinib in patients (pts) with previously treated locally advanced or metastatic cholangiocarcinoma (CCA). Ann Oncol. 2019, 30 (suppl_5):v876. 10.1093/annonc/mdz394.031

15. Doebele RC, Drilon A, Paz-Ares L, et al.: Entrectinib in patients with advanced or metastatic NTRK fusionpositive solid tumours: integrated analysis of three phase 1-2 trials. Lancet Oncol. 2020, 21:271-82. 10.1016/S1470-2045(19)30691-6

16. Drilon A, Laetsch TW, Kummar S, et al.: Efficacy of larotrectinib in TRK fusion-positive cancers in adults and children. N Engl J Med. 2018, 378:731-9. 10.1056/NEJMoa1714448

17. Abida W, Cyrta J, Heller G, et al.: Genomic correlates of clinical outcome in advanced prostate cancer . Proc Natl Acad Sci U S A. 2019, 116:11428-36. 10.1073/pnas.1902651116

18. de Bono JS, De Giorgi U, Rodrigues DN, et al.: Randomized phase II study evaluating Akt blockade with ipatasertib, in combination with abiraterone, in patients with metastatic prostate cancer with and without PTEN loss. Clin Cancer Res. 2019, 25:928-36. 10.1158/1078-0432.CCR-18-0981

19. Hyman DM, Smyth LM, Donoghue MT, et al.: AKT inhibition in solid tumors with AKT1 mutations . J Clin Oncol. 2017, 35:2251-9. 10.1200/JCO.2017.73.0143

20. André F, Ciruelos E, Rubovszky G, et al.: Alpelisib for PIK3CA-mutated, hormone receptor-positive advanced breast cancer. N Engl J Med. 2019, 380:1929-40. 10.1056/NEJMoa1813904

21. Hyman DM, Piha-Paul SA, Won H, et al.: HER kinase inhibition in patients with HER2- and HER3-mutant cancers. Nature. 2018, 554:189-94. 10.1038/nature25475

22. Mateo J, Chakravarty D, Dienstmann R, et al.: A framework to rank genomic alterations as targets for cancer 
precision medicine: the ESMO Scale for Clinical Actionability of molecular Targets (ESCAT). Ann Oncol. 2018, 29:1895-902. 10.1093/annonc/mdy263

23. Mosele F, Remon J, Mateo J, et al.: Recommendations for the use of next-generation sequencing (NGS) for patients with metastatic cancers: a report from the ESMO Precision Medicine Working Group. Ann Oncol. 2020, 31:1491-505. 10.1016/j.annonc.2020.07.014

24. Midha A, Dearden S, McCormack R: EGFR mutation incidence in non-small-cell lung cancer of adenocarcinoma histology: a systematic review and global map by ethnicity (mutMapII). Am J Cancer Res. 2015, 5:2892-911.

25. Mok TS, Cheng Y, Zhou X, et al.: Improvement in overall survival in a randomized study that compared dacomitinib with gefitinib in patients with advanced non-small-cell lung cancer and EGFR-activating mutations. J Clin Oncol. 2018, 36:2244-50. 10.1200/JCO.2018.78.7994

26. Soria JC, Ohe Y, Vansteenkiste J, et al.: Osimertinib in untreated EGFR-mutated advanced non-small-cell lung cancer. N Engl J Med. 2018, 378:113-25. 10.1056/NEJMoa1713137

27. Khozin S, Blumenthal GM, Jiang X, et al.: U.S. Food and Drug Administration approval summary: erlotinib for the first-line treatment of metastatic non-small cell lung cancer with epidermal growth factor receptor exon 19 deletions or exon 21 (L858R) substitution mutations. Oncologist. 2014, 19:774-9.

10.1634/theoncologist.2014-0089

28. Paz-Ares L, Tan EH, O'Byrne K, et al.: Afatinib versus gefitinib in patients with EGFR mutation-positive advanced non-small-cell lung cancer: overall survival data from the phase IIb LUX-Lung 7 trial. Ann Oncol. 2017, 28:270-7. 10.1093/annonc/mdw611

29. Dungo RT, Keating GM: Afatinib: first global approval. Drugs. 2013, 73:1503-15. 10.1007/s40265-013-01116

30. Ramalingam SS, Vansteenkiste J, Planchard D, et al.: Overall survival with osimertinib in untreated, EGFRmutated advanced NSCLC. N Engl J Med. 2020, 382:41-50. 10.1056/NEJMoa1913662

31. Mok TS, Wu YL, Ahn MJ, et al.: Osimertinib or platinum-pemetrexed in EGFR T790M-positive lung cancer . N Engl J Med. 2017, 376:629-40. 10.1056/NEJMoa1612674

32. Yang JC, Sequist LV, Geater SL, et al.: Clinical activity of afatinib in patients with advanced non-small-cell lung cancer harbouring uncommon EGFR mutations: a combined post-hoc analysis of LUX-Lung 2, LUXLung 3, and LUX-Lung 6. Lancet Oncol. 2015, 16:830-8. 10.1016/S1470-2045(15)00026-1

33. (20306620607032021). http://doi: 10.1016/S1470-2045(15)00026-1.

34. Peters S, Camidge DR, Shaw AT, et al.: Alectinib versus crizotinib in untreated ALK-positive non-small-cell lung cancer. N Engl J Med. 2017, 377:829-38. 10.1056/NEJMoa1704795

35. Camidge DR, Kim HR, Ahn MJ, et al.: Brigatinib versus crizotinib in advanced ALK inhibitor-naive ALKpositive non-small cell lung cancer: second interim analysis of the phase III ALTA-1L trial. J Clin Oncol. 2020, 38:3592-603. 10.1200/JCO.20.00505

36. Shaw AT, Bauer TM, de Marinis F, et al.: First-line lorlatinib or crizotinib in advanced ALK-positive lung cancer. N Engl J Med. 2020, 383:2018-29. 10.1056/NEJMoa2027187

37. Tong JH, Yeung SF, Chan AW, et al.: MET amplification and exon 14 splice site mutation define unique molecular subgroups of non-small cell lung carcinoma with poor prognosis. Clin Cancer Res. 2016, 22:304856. 10.1158/1078-0432.CCR-15-2061

38. Drilon A, Clark JW, Weiss J, et al.: Antitumor activity of crizotinib in lung cancers harboring a MET exon 14 alteration. Nat Med. 2020, 26:47-51. 10.1038/s41591-019-0716-8

39. Camidge DR, Otterson GA, Clark JW, et al.: Crizotinib in patients (pts) with MET-amplified non-small cell lung cancer (NSCLC): updated safety and efficacy findings from a phase 1 trial. J Clin Oncol. 2018, 36:9062. 10.1200/JCO.2018.36.15_suppl.9062

40. Fakih M, O'Neil B, Price TJ, et al.: Phase 1 study evaluating the safety, tolerability, pharmacokinetics (PK), and efficacy of AMG 510, a novel small molecule KRASG12C inhibitor, in advanced solid tumors. J Clin Oncol. 2019, 37:3003. 10.1200/JCO.2019.37.15_suppl.3003

41. Hallin J, Engstrom LD, Hargis L, et al.: The KRASG12C inhibitor MRTX849 provides insight toward therapeutic susceptibility of KRAS-mutant cancers in mouse models and patients. Cancer Discov. 2020, 10:54-71. 10.1158/2159-8290.CD-19-1167

42. Nogova L, Mattonet C, Scheffler M, et al.: Sorafenib and everolimus in patients with advanced solid tumors and KRAS-mutated NSCLC: a phase I trial with early pharmacodynamic FDG-PET assessment. Cancer Med. 2020, 9:4991-5007. 10.1002/cam4.3131

43. Nagasaka M, Li Y, Sukari A, Ou SI, Al-Hallak MN, Azmi AS: KRAS G12C Game of Thrones, which direct KRAS inhibitor will claim the iron throne?. Cancer Treat Rev. 2020, 84:101974. 10.1016/j.ctrv.2020.101974

44. Veenstra DL, Mandelblatt J, Neumann P, Basu A, Peterson JF, Ramsey SD: Health economics tools and precision medicine: opportunities and challenges. Forum Health Econ Policy. 2020, 23: 10.1515/fhep-20190013

45. Weymann D, Pataky R, Regier DA: Economic evaluations of next-generation precision oncology: a critical review. JCO Precis Oncol. 2018, 2:10.1200/PO.17.00311

46. Tan AC, Lai GG, Tan GS, et al.: Utility of incorporating next-generation sequencing (NGS) in an Asian nonsmall cell lung cancer (NSCLC) population: incremental yield of actionable alterations and costeffectiveness analysis. Lung Cancer. 2020, 139:207-15. 10.1016/j.lungcan.2019.11.022

47. Lindeman NI, Cagle PT, Beasley MB, et al.: Molecular testing guideline for selection of lung cancer patients for EGFR and ALK tyrosine kinase inhibitors: guideline from the College of American Pathologists, International Association for the Study of Lung Cancer, and Association for Molecular Pathology. J Mol Diagn. 2013, 15:415-53. 10.1016/j.jmoldx.2013.03.001

48. Shi Y, Au JS, Thongprasert S, et al.: A prospective, molecular epidemiology study of EGFR mutations in Asian patients with advanced non-small-cell lung cancer of adenocarcinoma histology (PIONEER). J Thorac Oncol. 2014, 9:154-62. 10.1097/JTO.0000000000000033

49. Steuten L, Goulart B, Meropol NJ, Pritchard D, Ramsey SD: Cost effectiveness of multigene panel sequencing for patients with advanced non-small-cell lung cancer. JCO Clin Cancer Inform. 2019, 3:1-10. 10.1200/CCI.19.00002 